\title{
Fully Automatic 3-D-TEE Segmentation for the Planning of Transcatheter Aortic Valve Implantation
}

\author{
Sandro Queirós*, Alexandros Papachristidis, Pedro Morais, Konstantinos C. Theodoropoulos, \\ Jaime C. Fonseca, Mark J. Monaghan, João L. Vilaça, and Jan D’hooge
}

\begin{abstract}
A novel fully automatic framework for aortic valve (AV) trunk segmentation in three-dimensional (3-D) transesophageal echocardiography (TEE) datasets is proposed. The methodology combines a previously presented semiautomatic segmentation strategy by using shapebased B-spline Explicit Active Surfaces with two novel algorithms to automate the quantification of relevant AV measures. The first combines a fast rotation-invariant 3-D generalized Hough transform with a vessel-like dark tube detector to initialize the segmentation. After segmenting the AV wall, the second algorithm focuses on aligning this surface with the reference ones in order to estimate the short-axis (SAx) planes (at the left ventricular outflow tract, annulus, sinuses of Valsalva, and sinotubular junction) in which to perform the measurements. The framework has been tested in 20 3-D-TEE datasets with both stenotic and nonstenotic AVs. The initialization algorithm presented a median error of around $3 \mathrm{~mm}$ for the AV axis endpoints, with an overall feasibility of $90 \%$. In its turn, the SAx detection algorithm showed to be highly reproducible, with indistinguishable results compared with the variability
\end{abstract}

Manuscript received September 10, 2016; accepted October 7, 2016. Date of publication October 13, 2016; date of current version July 15, 2017. This work was supported by the project "ON.2 SR\&TD Integrated Program (Norte-07-0124-FEDER-000017)" cofunded by the Programa Operacional Regional do Norte (ON.2- O Novo Norte), Quadro de Referência Estratégico Nacional, through Fundo Europeu de Desenvolvimento Regional. The work of S. Queirós and P. Morais was supported by the FCT-Fundação para a Ciência e a Tecnologia and the European Social Found through the Programa Operacional Capital Humano in the scope of the Ph.D. Grants SFRH/BD/93443/2013 and SFRH/BD/95438/2013, respectively. J. L. Vilaça and J. D'hooge are joint last authors. Asterisk indicates corresponding author.

*S. Queirós is with the ICVS/3B's-PT Government Associate Laboratory and the Algoritmi Center, University of Minho, 4710-057 Braga, Portugal, and also with the Lab on Cardiovascular Imaging and Dynamics, Department of Cardiovascular Sciences, KU Leuven, Leuven 3000, Belgium (e-mail: sandroqueiros@med.uminho.pt).

A. Papachristidis, K. C. Theodoropoulos, and M. J. Monaghan are with the King's College Hospital NHS Foundation Trust, London, UK.

P. Morais is with the ICVS/3B's-PT Government Associate Laboratory, University of Minho, Portugal, with the Lab on Cardiovascular Imaging and Dynamics, Department of Cardiovascular Sciences, KU Leuven, Belgium, and with the INEGI, Faculty of Engineering , University of Porto, Portugal.

J. L. Vilaça is with the ICVS/3B's-PT Government Associate Laboratory and also with the DIGARC-Polytechnic Institute of Cávado and Ave, Portugal.

J. C. Fonseca is with the Algoritmi Center, School of Engineering, University of Minho, Guimarães, Portugal.

J. D'hooge is with the Lab on Cardiovascular Imaging and Dynamics, Department of Cardiovascular Sciences, KU Leuven, Leuven, Belgium.

Digital Object Identifier 10.1109/TBME.2016.2617401 found between the experts' defined planes. Automatically extracted measures at the four levels showed a good agreement with the experts' ones, with limits of agreement similar to the interobserver variability. Moreover, a validation set of $\mathbf{2 0}$ additional stenotic AV datasets corroborated the method's applicability and accuracy. The proposed approach mitigates the variability associated with the manual quantification while significantly reducing the required analysis time (12 s versus 5 to $10 \mathrm{~min}$ ), which shows its appeal for automatic dimensioning of the AV morphology in 3-D-TEE for the planning of transcatheter AV implantation.

Index Terms-Aortic valve (AV) segmentation, automatic initialization, fully automatic quantification, transcatheter aortic valve implantation, 3-D transesophageal echocardiography (TEE).

\section{INTRODUCTION}

A ORTIC stenosis (AS), a condition in which the aortic valve (AV) calcifies and obstructs the flow from the left ventricle (LV) to the aorta, is the most common acquired valvular heart disease in developed countries [1]. Nowadays, high-risk patients with symptomatic, severe AS are indicated for transcatheter AV implantation (TAVI) [1]. During this intervention, a prosthetic valve is deployed in the aortic annulus (AoA) to replace the patient's original malfunctioning valve. To successfully deploy the prosthesis, pre- and peri-procedural imaging are paramount [2], [3]. During the planning stage, clinicians must assess the feasibility of the access site, review the AV morphology and function, and perform several anatomical measurements [2][4]. Among these, AoA sizing is mandatory for prosthesis sizing [2], [4]-[6]. Measurements at other levels, namely LV outflow tract (LVOT), sinuses of Valsalva (SoV), and sinotubular junction (STJ), can also be required for a proper treatment planning [5]-[7]. To this end, both multidetector computed tomography (MDCT) and three-dimensional (3-D) transesophageal echocardiography (TEE) have been used [2], [6], [8] with comparable results [6], [9]-[11].

To help clinicians assess the AV morphology, several methods have already been proposed to (semi-) automatically segment it in MDCT [12]-[17], 2-D-TEE [18]-[20] and, more recently, 3-D-TEE datasets [12], [21]-[24]. Besides being possible to separate these studies based on their target modality, they can also be divided according to the methodology used (machine learning [12], [15], [17], deformable models [13], [21], [22], [24], 


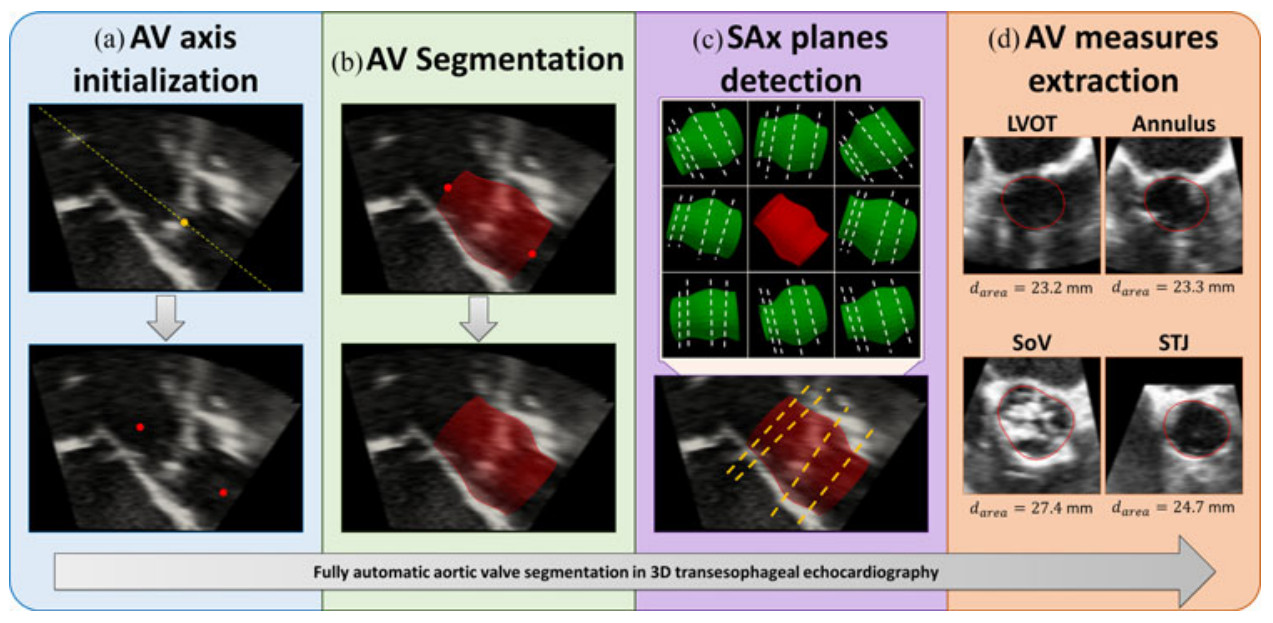

Fig. 1. Overview of the proposed fully automatic AV tract segmentation framework.

among others) or the required level of user interaction (fully automatic [12], [15]-[17], [21], [22], semi-automatic [18]-[20], [23], [24] or interactive [14]).

Among 3-D-TEE methodologies, Ionasec et al. [12] proposed the first fully automatic algorithm for AV segmentation. Their framework uses marginal space learning (MSL) and steerable features to locate the valve's bounding box, which is then segmented based on the MSL and learned boundary detectors. On its turn, Weber et al. [22] presented an adapted 3-D generalized Hough transform (GHT) to detect the AV, followed by a deformable model strategy with optimal boundary detection functions and shape prior information. Also targeting a fully automatic segmentation, Bersvendsen et al. [21] proposed an algorithm based on the state-space estimation of a subdivision deformable surface. Although being fully automatic, no AV detection algorithm was required since the authors claim that the initialization can be derived directly from the ultrasound machine (recorded roll angle). Finally, Pouch et al. [23] proposed a semiautomatic methodology to extract the AV root and leaflets using multi-atlas label fusion and template-based branching medial modeling. Their strategy requires five initial landmark points to register the target image with each atlas.

Recently, we proposed a semiautomatic algorithm for AV trunk segmentation in 3-D-TEE data by using the shape-based B-spline Explicit Active Surfaces (BEAS) [24]. The method was shown to be accurate, robust, and computationally efficient, taking less than one second to segment the entire AV wall from the ascending aorta (AscAo) to the LVOT with an average accuracy of $0.78 \mathrm{~mm}$. Moreover, semiautomatically derived clinical measurements showed an excellent agreement with the experts' ones. Nevertheless, the strategy required two user-defined points at the AV lumen to initialize the segmentation: one at the AscAo and another at the LVOT. At the end, clinical measures were extracted by cutting the AV surface at the short-axis (SAx) planes identified by an expert.

Focusing on mitigating the method's user dependence, in this study, we propose to fully automatize our AV trunk segmentation algorithm by adding two automatic routines (see Fig. 1). The first one focuses on identifying the AscAo and LVOT points required to initialize the original segmentation strategy. The second one automatically aligns the resulting segmented AV wall with the reference surfaces in order to estimate the SAx planes of interest (LVOT, AoA, SoV, and STJ) in which to perform the measurements. Overall, the key novelties introduced are fourfold:

1) a novel fully automatic methodology for AV trunk segmentation and quantification in 3-D-TEE datasets;

2) a fast AV centroid detection technique through an adapted rotation-invariant 3-D GHT;

3) a novel method for AV axis identification using a vessellike dark tube detector; and

4) a SAx plane estimator based on the iterative closest point (ICP) algorithm and known AV reference surfaces.

The proposed fully automatic methodology was assessed against manual annotations and measurements in 40 3-D-TEE datasets, including 30 patients with AS and 10 with nonstenotic AV.

This paper is structured as follows. In Section II, a description of the proposed fully automatic framework is presented. Section III presents the validation experiments and corresponding results. In Section IV, we discuss the performance of the proposed framework, and compare against the previous semiautomatic strategy and other state-of-the-art methods. The main conclusions are given in Section V.

\section{METHODS}

\section{A. Overview}

The proposed framework aims to automatically segment the AV tract (from AscAo to LVOT) in the 3-D-TEE datasets, allowing us to assess its morphology and extract clinical measures.

The proposed methodology is divided in four main stages:

1) a fast rotation-invariant 3-D GHT and a vessel-like dark tube detector are combined to automatically estimate the AV centroid and its main axis [yellow dot and dashed line in Fig. 1(a), Section II-B], from which the 2 axis points (at the AscAo and LVOT) can then be estimated (red dots); 




Fig. 2. Overview of the proposed initialization strategy. (a) Candidate AV centroid (red dot) is found using a fast rotation-invariant 3-D GHT applied to an edge map. (b) Using the candidate centroids, the best fitting axis (red dashed line) is found by searching (along both elevation and azimuth angles) for a dark, vessel-like tube structure. (c) By initializing a cylindrical surface into the estimated axis, a threshold-based BEAS evolution is applied, refining the AV axis and centroid (red dashed line and dot, respectively). (d) The image intensity sum inside a square is computed along the axis, with its maximum matching the leaflets tissue near the annulus (blue dot). Using a heuristic rule (blue dashed arrows), the two endpoints (AscAo and LVOT) are positioned (green dots).

2) using the identified axis, the previously proposed segmentation method [24] is applied to accurately delineate the AV wall [see red surface in Fig. 1(b), Section II-C];

3 ) the ICP algorithm is used to align our automatically segmented surface with several reference ones (green surfaces, with known SAx planes in white dashed lines), allowing us to estimate the SAx planes of interest [orange dashed lines in Fig. 1(c), Section II-D]; and

4) ultimately, the AV tract surface is cut at each SAx plane and the obtained contour used to extract the relevant diameters [see Fig. 1(d), derived from area, perimeter or maximum/minimum lengths].

\section{B. Automatic Axis Initialization}

In order to define an initial AV axis to start the segmentation approach, a four-step initialization strategy is proposed (see Fig. 2):

1) a fast rotation-invariant 3-D GHT is applied to detect candidate centroids for the AV dataset under analysis (Section II-B1);

2) a vessel-like dark tube detector is used to estimate the AV main axis orientation (Section II-B2);

3) a threshold-based BEAS [25] is then applied to refine the orientation of the estimated $\mathrm{AV}$ axis (Section II-B3); and

4) an intensity-based method detects the leaflets' tissue, to which a heuristic rule is applied to place the two initial AV axis points: one at the AscAo and another at the LVOT (Section II-B4).

1) Fast Rotation-Invariant 3-D GHT: The GHT [26] is a robust method to detect arbitrary shapes in an image undergoing geometric transformations. Its original formulation suffers from high memory and computational burden, which hampers its use when rotation and scaling parameters are unknown (which requires testing exhaustive mutual combinations of

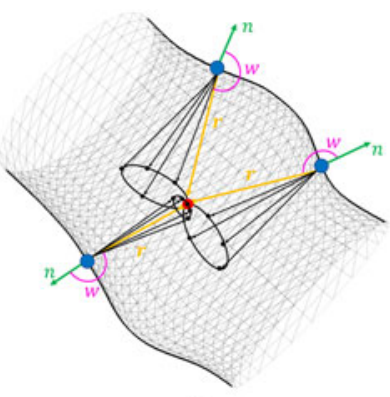

(a)

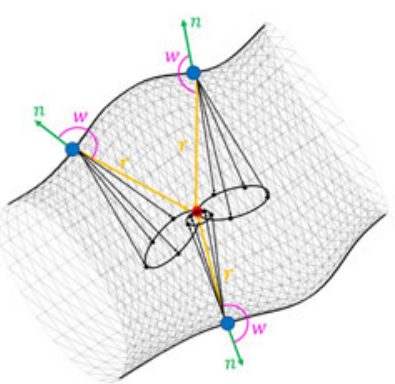

(b)
Fig. 3. Principle of the rotation-invariant 3-D GHT. The angle $w$ (given wrt. the surface points' normal, $n$ ) is invariant to $3-\mathrm{D}$ rotation. Each reference pair $[r, w]$ defines a circle in space, with all points voting for the centroid.

seven parameters). In medical applications, rotation is usually considered to be known a priori (or its variability small enough for such assumption [27]), with scaling being introduced by testing a short range of possible values. For the specific case of the $\mathrm{AV}$ in the 3-D-TEE zoomed datasets, although scaling has a small variation, the rotation parameters are quite variable. Indeed, the $\mathrm{AV}$ can be rotated up to $90^{\circ}$ in both elevation and azimuth angles. Thus, a rotation-invariant 3-D GHT is preferred, and is here applied by using the modified approach proposed by Rozhentsov et al. [28].

In the GHT approach, during learning, the description of the shape under analysis is encoded in a reference table (known as R-table). Rozhentsov et al. [28] propose to describe its entries as vectors pointing from a boundary point to the shape centroid and encoded by the angle $w$ (between the normal at the surface point and the direction to the centroid) and distance $r$ (between point and centroid), both invariant to rotation (see Fig. 3). During testing, for each sample point (in a total of $N_{\text {samples }}$ ) and each 


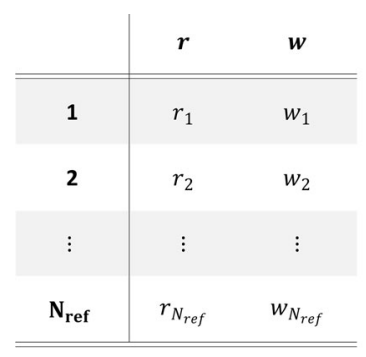

(a) (b)

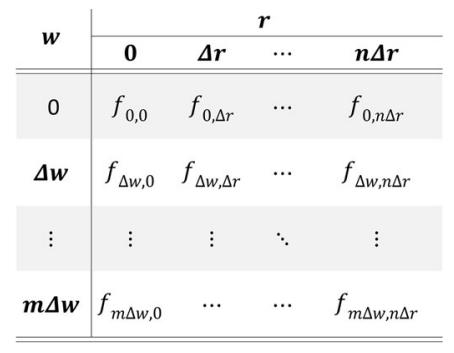

Fig. 4. (a) R-table of the original implementation in [28]. (b) Proposed rearranged R-table, with both $w$ and $r$ values discretized (into $m$ and $n$ angular and distance values, respectively) and corresponding frequency of each pair.

R-table pair $\left(\left[r_{i}, w_{i}\right], i=1, \ldots, N_{\text {ref }}\right)$, the possible centroid positions are estimated and voted into an accumulator. Note that each reference pair defines a circle in the 3-D space, in which every point can be the true centroid (see Fig. 3). Finally, the accumulator is smoothed and its highest peak found [see Fig. 2(a)], corresponding to the estimated shape centroid.

In our implementation, we modified the above-mentioned strategy as follows.

First, since the same strategy should be used to determine boundary points in the training and testing phases, a 3-D Canny edge detector [29] was used [see Fig. 2(a)]. In the training stage, as the AV surface is known, only edges close to its boundaries are considered as reference points (within $4 \mathrm{~mm}$ distance) and included into the R-table. During testing, all edge points in the TEE field-of-view (FOV) are used as test points. Note that, at the initialization stage, all images were resampled to a voxel resolution of $\sim 1.0 \times 1.0 \times 1.0 \mathrm{~mm}^{3}$ to decrease the method's computational burden.

Second, we reduced the computational complexity of the method by rearranging its R-table. Instead of considering each reference point independently, we group them by discretizing both $w$ and $r$ values, and finding the frequency of each pair among the reference points (see Fig. 4). During testing, each reference pair votes to the accumulator by summing its own frequency value, $f_{\left(r_{i}, w_{i}\right)}$. Such simplification reduces the number of reference pairs to be tested per edge point, thus significantly decreasing the method's computational time. In this study, ten discrete angular values were considered and the distance values rounded to even numbers, leading to a 2000 times faster voting process.

Third, since the method is rotation invariant, there is no need for shape superposition and distinct training datasets can be combined by simply fusing their respective R-tables. In this way, the combined R-table will intrinsically embed the variability seen in the structure to be detected (the AV), both in terms of scaling and shape.

Finally, after smoothing the accumulator (with a cubic element of $7 \mathrm{~mm}$ ) and in order to increase the method's robustness, $N$ high-probability centroid candidates are retained (ten in this study), which will be included in the following stage to determine the AV axis' orientation.

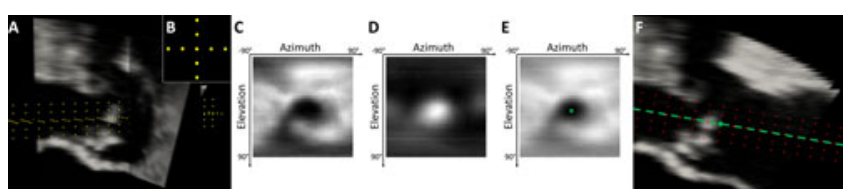

Fig. 5. (A) Tubular structure (yellow dots) used in the proposed detector, and its cross section (B). Example (C) intensity map, (D) vesselness map, and (E) combined map as a function of azimuth and elevation angles. (F) Example best fitting tubular structure (red dots) for an AS dataset, and associated AV centroid and orientation found (green dot and dashed line, respectively).

2) Vessel-Like Dark Tube Detector: Taking into account the valve's estimated position within the image FOV, we now ought to find its orientation. Hereto, two a priori knowledge of the AV shape and appearance in the 3-D-TEE datasets are exploited: 1) the AV is a large vessel, i.e., a tubular structure; and 2) it has a dark lumen surrounded by bright walls. In this sense, we propose a novel fast vessel-like dark tube detector.

The proposed detector exhaustively tests possible orientations (by varying the elevation and azimuth angles) of a tubular structure [see Fig. 5(A) and (B)] centered on a candidate centroid. The structure's outer diameter was chosen in order to always be smaller than the normal AV lumen's diameter, having been empirically set to $10 \mathrm{~mm}$. In this study, the ranges mentioned above $\left( \pm 90^{\circ}\right)$ were considered for the possible orientations, with $5^{\circ}$ intervals. For each orientation, the structure's points are positioned on the top of the intensity image, and the corresponding values averaged. Such process creates an intensity map [as a function of both elevation and azimuth angles, see Fig. 5(C)], where the minimum value corresponds to the darkest tubular structured centered in the candidate centroid. To embed the AV's vessel-like shape in the detector, the vesselness filter introduced by Frangi et al. [30] is used, and the same mapping process repeated by averaging the vessel-enhanced 3-D image values. In this case, the maximum value corresponds to the most vessel-like tubular structure centered in the candidate centroid [Fig. 5(D)]. By summing both maps (after inverting the vesselness one), we obtain a map [Fig. 5(E)] in which the minimum value corresponds to the most dark, vessel-like tubular structure centered in the candidate centroid under analysis. This process is repeated for the $N$ candidate centroids retained in Section II-B1, and their minimum value, and corresponding orientation, gathered. Finally, the candidate with the lowest minimum value simultaneously defines the best fitting orientation and centroid position for the AV [see Fig. 5(E) and (F)].

3) AV Axis Refinement: Due to the tubular structure's fixed size, the AV axis orientation estimated in Section II-B2 can be, in some cases, oblique with respect to the valve's true axis [particularly at the AscAo side, due to its larger diameter-see Fig. 5(D)]. Therefore, we propose to refine the estimated orientation using the fast threshold-based BEAS algorithm [25]. Hereto, the idea is to start with a fixed size cylindrical surface around the estimated AV axis (with $20 \mathrm{~mm}$ length to each side of the centroid) and evolve it using a threshold-based evolution [see Fig. 2(C)]. Thus, the surface will radially grow if the intensity of the surface point under 
analysis is higher than a predefined threshold. On the contrary, when lower, the surface will shrink. In this study, the threshold was set as the average value between the maximum and minimum gray values seen in the input image. Since no energy is explicitly defined for such threshold-based evolution, a fixed number of iterations (25 in this study) are applied to evolve the surface. In the end, the surface's refined centroid and axis orientation can thus be extracted [red dashed line in Fig. 2(c)]. Note, however, that since the AV length used is fixed and not covering the region between the AscAo and LVOT only, the obtained surface does not accurately capture the AV wall.

4) AV Axis Endpoints Detection: At this stage, and due to the requirements of the segmentation algorithm [24], the two axis endpoints need to be estimated. Nonetheless, since these are not associated with the well-defined anatomical landmarks, their direct estimation is challenging, particularly in the AS cases. Thus, we instead propose to estimate the leaflets' coarse position and apply a heuristic rule to place the two axis endpoints.

To this end, a square region is translated along the estimated $\mathrm{AV}$ axis and the average intensity within is computed. Note that the region's size $\left(24 \times 24 \mathrm{~mm}^{2}\right.$ in this study) was set according to the average AoA diameter [24]. Since the leaflets are the brightest structures within the AV lumen (and more dense closer to the hinge points), their location will correspond to the maximum average value found [see Fig. 2(d)]. Based on the average AV length (from AscAo to LVOT) computed from the statistical shape model (SSM) in [24] and its relation to the leaflets tissue, both AscAo and LVOT endpoints are positioned by translating the point found by 20 and $13 \mathrm{~mm}$ to each side, respectively [see Fig. 2(d)].

\section{AV Segmentation}

After estimating the two AV axis endpoints, our previously proposed shape-based BEAS segmentation method [24] is applied to accurately delineate the AV wall [see Fig. 1(b)].

Overall, a 3-D cylindrical formulation of the BEAS framework is used through a dual-stage energy evolution process, comprising a threshold-based coarse segmentation and a localized region-based refinement. Hereto, intensity- and shape-based features are combined to increase accuracy and robustness. Shape-prior information is included using a profilebased SSM and embedded in BEAS through two regularization terms: one confining the segmented AV profiles to shapes familiarly seen in the SSM (hard regularization) and another penalizing according to the profile's degree of likelihood (soft regularization). The hybrid energy functional takes the advantage of the intensity data in regions with strong image content, while complementing it with shape knowledge in regions with nearly absent image data. The latter is particularly relevant in AS due to the acoustic shadowing artifacts associated with the calcifications. Note that this methodology also includes an AV axis reinitialization strategy, focused on reducing the effect of suboptimal endpoints' positioning. For more details on the segmentation strategy, the reader is kindly referred to [24].

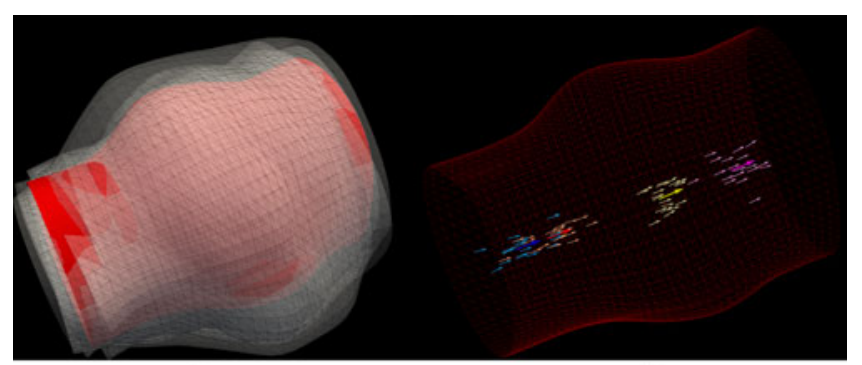

(a)

(b)

Fig. 6. (a) Reference surfaces (in white) after ICP rigid alignment with target AV surface (in red). (b) Candidate planes (represented as vectors with given position and direction) and resulting average SAx plane at different levels of interest (cyan/blue: LVOT; orange/red: annulus; light/dark yellow: SoV; pink/magenta: STJ, for candidate vectors and average one, respectively)

\section{Automatic SAx Planes Detection}

In order to estimate the SAx planes in which to measure the $\mathrm{AV}$, we propose to apply the ICP algorithm to rigidly align the segmented AV surface with reference ones [see Fig. 6(a)]. To increase the algorithm's robustness, the surfaces' main axes are used to prealign them prior to the ICP. Since these reference surfaces are linked to corresponding ground-truth SAx planes (at the four levels of interest-LVOT, AoA, SoV, and STJ), it is possible to estimate the location and normal direction of these anatomical planes in the target dataset. By performing the alignment with multiple references, several candidate planes are estimated for each level, and their locations and normal vectors can be averaged [see Fig. 6(b)]. In this sense, misplacement due to suboptimal alignment with one particular reference surface (with a more distinct 3-D shape) is alleviated, and the resulting SAx planes still robustly extracted [see Fig. 1(c)].

At this point, the surface is cut using each plane, and the obtained contours used to extract the measurements [see Fig. 1(d)].

\section{EXPERIMENTS AND RESULTS}

\section{A. Data Description}

The assessment of the proposed framework was carried out on 403 -D-TEE datasets of patients affected by various cardiovascular diseases. These images were acquired during clinical cardiology routine and retrospectively provided by the King's College Hospital, London, U.K. For the present experiments, these datasets were divided into three groups: 10 nonstenotic and 10 stenotic AV, plus 20 additional stenotic AV cases. During the algorithm testing, the two first group of cases were used for training and testing in a leave-one-out strategy. The third group was used as a validation set (see Section III-G). The images were acquired from the mid-esophageal position using 3-D zoom mode (as recommended in the current guidelines [8]) and included the LVOT, AV, and aortic root. All images were recorded with a Philips Epiq 7 ultrasound machine and an X7$2 t$ transducer (Philips Healthcare, Andover, MA). At least one complete cardiac cycle was captured in a sequence of 9-38 volumes, with an image resolution and size from 0.21 to $0.31 \mathrm{~mm}$ and $228 \times 231 \times 208$ to $345 \times 335 \times 208$ voxels, respectively. 
TABLE I

Performance for the Different Stages of the Initialization Algorithm [5th Percentile, Median and 95th Percentile]

\begin{tabular}{|c|c|c|c|c|c|c|}
\hline \multicolumn{2}{|l|}{ Stage } & \multirow{2}{*}{$\frac{\text { AV centroid }(\mathrm{mm})}{\left[\begin{array}{lll}1.01 & 2.73 & 7.13\end{array}\right]}$} & \multirow{2}{*}{$\frac{\mathrm{AV} \text { axis }\left(^{\circ}\right)}{-}$} & \multirow{2}{*}{$\begin{array}{c}\text { AscAo endpoint (mm) } \\
-\end{array}$} & \multirow{2}{*}{$\frac{\text { LVOT endpoint }(\mathrm{mm})}{-}$} & \multirow{2}{*}{$\frac{\text { Cumulative feasibility }}{20 / 20(100 \%)}$} \\
\hline 3-D GHT & & & & & & \\
\hline Tube detector & & {$\left[\begin{array}{llll}0.63 & 3.11 & 6.27\end{array}\right]$} & {$\left[\begin{array}{llll}2.89 & 6.04 & 12.14\end{array}\right]$} & - & - & $20 / 20(100 \%)$ \\
\hline Axis refinement & & {$\left[\begin{array}{llll}0.44 & 1.65 & 2.54\end{array}\right]$} & {$\left[\begin{array}{llll}0.88 & 4.84 & 13.77\end{array}\right]$} & - & - & $19 / 20(95 \%)$ \\
\hline Endpoints' estimation & $\mathrm{P} 2 \mathrm{Pt}$ & - & - & [ 1.05 3.23 7.69$]$ & {$\left[\begin{array}{llll}1.43 & 3.11 & 7.02\end{array}\right]$} & $18 / 20(90 \%)$ \\
\hline & $\mathrm{P} 2 \mathrm{Pl}$ & - & - & {$\left[\begin{array}{llll}0.03 & 1.86 & 5.45\end{array}\right]$} & {$\left[\begin{array}{llll}0.11 & 1.36 & 4.84\end{array}\right]$} & \\
\hline
\end{tabular}

\section{B. Manual Analysis}

The ground truth was obtained through an annotation process performed by experts using custom noncommercial software, Speqle3D [31]. For each dataset, both AV axis, surface and clinical measures were extracted at a mid-systolic frame. To start, the user defined the $\mathrm{AV}$ axis by identifying two points (at the AscAo and at the LVOT). Then, the user manually traced 15-21 planes perpendicular to this axis. The manually annotated scattered points were then converted into the cylindrical space (using the defined axis), interpolated to a regular grid and converted back into the Cartesian space, thus creating spatially consistent 3-D surfaces. Regarding the reference measures, the cardiology expert freely explored the volume by using three orthogonal planes and set four SAx planes in which to perform them (as recommended in [4]), corresponding to the LVOT, AoA, SoV, and STJ planes. In each plane, the target structure was manually traced and perimeter-derived diameters were extracted (henceforward referred as Manual1).

To establish the reproducibility of the SAx planes definition, manual tracings, and measures, a second expert reanalyzed each dataset of the training set (i.e., ten nonstenotic and ten AS cases). A completely independent analysis was performed (i.e., defining four new SAx planes and extracting the perimeter-derived diameters, referred as Manual2), thus representing the interobserver variability seen in clinical routine.

\section{AV Axis Initialization Performance}

The performance of the initialization algorithm was assessed by comparing the results of each stage against expert's annotations. The centroid error was measured as the minimum Euclidean distance between the identified point (at the different stages) and the valve's central axis (given by the two expertdefined endpoints). The AV axis was assessed by computing the angle between the estimated axis and the expert-defined one. The accuracy of the identified endpoints was computed by calculating the Euclidean distance from the estimated points and each expert's endpoints. Both point-to-point ( $\mathrm{P} 2 \mathrm{Pt})$ and pointto-plane (P2Pl, i.e., distance from the identified point to the plane centered in the axis's endpoint and whose normal is equal to the AV axis) were computed. P2Pl distance was considered to distinguish transversal and longitudinal misplacements. Indeed, the segmentation should be accurate when an oblique or parallel axis is given (details in [24]), while longitudinal misplacements might have a higher impact on its performance. Note that a leave-one-out strategy was used for the 3-D GHT training, removing the reference edge voting points gathered from the case under analysis.
Table I summarizes the performance for the four stages of the initialization algorithm, with the cumulative feasibility of the method (after each stage) also presented. The latter was assessed by visual inspection of the initialization result for each dataset. Overall, the AV initialization strategy presented a feasibility of $90 \%$, only obtaining suboptimal results in two cases: one due to the axis identification pipeline (2nd and 3rd stages) and another due to the endpoints' estimation. The remaining cases were accurately initialized, with a median endpoints' P2Pt error of approximately $3 \mathrm{~mm}$ (1.86 and $1.36 \mathrm{~mm}$ P2Pl distance for the AscAo and LVOT, respectively). Note that the proposed multistage algorithm allows the continuous improvement of the estimated centroid and AV axis (see Table I), thus allowing us to partially recover from suboptimal results of precedent stages.

\section{SAx Planes Detection Performance}

1) Reproducibility Experiment: In order to evaluate the SAx planes detection algorithm, we started verifying the method's robustness and reproducibility against the endpoints' positioning and resultant segmented AV surface. In this sense, for each dataset, five randomly positioned endpoints (around the ones manually identified by the expert) were generated. Note that the original AscAo and LVOT endpoints were, in average, respectively translated $3.73 \pm 2.19$ and $3.51 \pm 2.28 \mathrm{~mm}$ (maximum displacement near $10 \mathrm{~mm}$ ). Each endpoints' pair was used as initialization for the segmentation algorithm [24], while subsequently applying the proposed SAx plane detection algorithm (using a leave-one-out strategy). Each set of estimated planes was then compared against the planes positioned by the first expert. In this sense, the P2Pl distance from the automated planes' centroid and the reference plane was computed. Moreover, the angles between the automated and reference planes' normals were also calculated. In total, 100 sets of SAx planes (each set containing the four planes at the LVOT, AoA, SoV, and STJ) were evaluated.

Overall, the identified LVOT, AoA, SoV, and STJ planes presented a P2Pl error of [ $\left.\begin{array}{lll}0.07 & 1.55 & 5.19\end{array}\right] \mathrm{mm}$ (5th percentile, median, and 95th percentile), [ [ $\left.\begin{array}{llll}0.11 & 1.79 & 4.80\end{array}\right] \mathrm{mm},\left[\begin{array}{lll}0.14 & 1.27\end{array}\right.$ 4.28 mm, [ $\left.\begin{array}{llll}0.14 & 1.46 & 3.79\end{array}\right] \mathrm{mm}$, respectively. Regarding the planes' normal, the angular error was [1.40 5.66 12.24 $]^{\circ},[1.55$ $5.6511 .55]^{\circ},\left[\begin{array}{lll}1.55 & 6.88 & 13.06\end{array}\right]^{\circ}$, and [1.84 6.6912 .37$]^{\circ}$, respectively. In order to assess its effect on the measurements, the Bland-Altman plots [32] (see Fig. 7) of the measured perimeterderived diameters were computed for the four levels. Hereto, the average diameter for each patient and at each level was considered as reference, while assessing the corresponding standard deviation (SD) for the 100 cases. Importantly, narrow limits of 


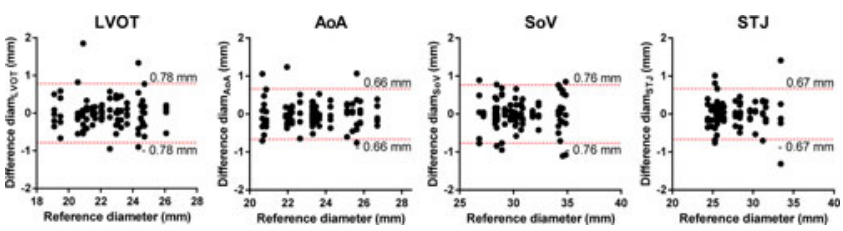

Fig. 7. Bland-Altman plots obtained in the reproducibility test for perimeter-derived diameters at the four SAx planes.

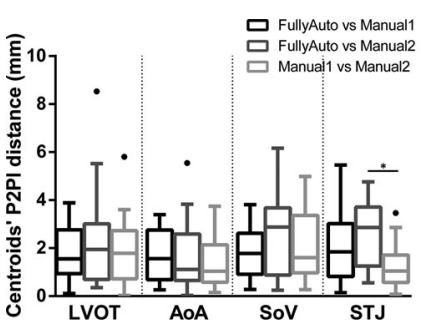

(a)



(b)
Fig. 8. Boxplots of (a) centroids' P2PI distance and (b) normals' angular difference between fully automatic SAx planes and manually identified ones. The interobserver variability is also depicted. Outliers are shown as black dots. ${ }^{*} p<0.05$, in a Mann-Whitney test between results.

agreement $(\mathrm{LOA}, 1.96 * \mathrm{SD})$ were obtained, showing the measurements reproducibility when combining both segmentation and SAx planes detection algorithms.

2) Accuracy Test With Fully Automatic Pipeline: In this experiment, the estimated SAx planes were evaluated after applying the fully automatic pipeline (i.e., automatic axis initialization, wall segmentation, and SAx planes detection). Since the initialization showed a feasibility of $90 \%$, only the successfully initialized datasets (18/20) were considered. The estimated planes were compared against the SAx planes manually defined by both experts (Manual1 and Manual2). For comparative purposes, the interobserver variability on the SAx planes definition (for the same 18 cases) was also calculated. In order to check for differences between the automatic strategy and the experts, the Mann-Whitney test was used (a $p$-value $<0.05$ was considered statistically significant).

Fig. 8 illustrates the obtained results, both in terms of centroids' P2Pl distance and normals' angular difference (see calculation details in Section III-D1). Overall, similar errors were obtained when compared with the reproducibility test previously performed (semiautomatic strategy, Section III-D1), with median distances lower than $2 \mathrm{~mm}$ and median angular differences between $6^{\circ}$ and $9^{\circ}$. Interestingly, these were also similar to the variability observed between experts, with no statistically significant differences (except for the STJ centroid when against the 2 nd observer). Ultimately, it shows the robustness and accuracy of the proposed automatic algorithm for the identification of the SAx planes of interest.

\section{E. Computational Time}

In what concerns its computational time, the fully automatic methodology took, on average, $11.9 \pm 2.4 \mathrm{~s}$ (MATLAB code running on a Intel(R) Core (TM) i7 $\mathrm{CPU}$ at $2.8 \mathrm{GHz}$, with
TABLE II

Agreement Between Fully Automatic and Manual Diameters (IN MM, FA/MANUAL1, AND FA/MANUAL2), AND INTEROBSERVER VARIABILITY (MANUAL1/MANUAL2)

\begin{tabular}{|c|c|c|c|}
\hline & LVOT & AoA & STJ \\
\hline \multicolumn{4}{|c|}{ FA/Manual1 } \\
\hline $9 \mathrm{NS}$ & $0.36 \pm 1.26^{\mathrm{c}}$ & $0.92^{\mathrm{ab}} \pm 1.06-1.03^{\mathrm{ab}} \pm 0.94$ & $0.94 \pm 1.44$ \\
\hline $9 \mathrm{~S}$ & $0.76 \pm 1.62$ & $0.61 \pm 1.55-1.76^{\mathrm{ab}} \pm 1.94$ & $1.69^{\mathrm{a}} \pm 1.52$ \\
\hline All & $0.56 \pm 1.42$ & $0.77^{\mathrm{a}} \pm 1.29-1.40^{\mathrm{ab}} \pm 1.53$ & $1.31^{\mathrm{a}} \pm 1.49$ \\
\hline \multicolumn{4}{|c|}{ 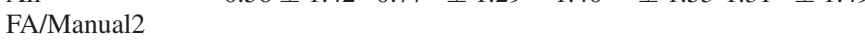 } \\
\hline 9NS & $0.35 \pm 0.95$ & $0.61 \pm 1.56-0.33^{\mathrm{b}} \pm 0.77$ & $1.89^{\mathrm{a}} \pm 0.72$ \\
\hline $9 \mathrm{~S}$ & $0.53 \pm 2.21$ & $0.99 \pm 1.59-1.06^{\mathrm{b}} \pm 2.53$ & $2.02^{\mathrm{ab}} \pm 1.15$ \\
\hline All & $0.44 \pm 1.65$ & $0.80^{\mathrm{a}} \pm 1.54-0.69^{\mathrm{b}} \pm 1.85^{\mathrm{c}}$ & $1.95^{\mathrm{ab}} \pm 0.93$ \\
\hline \multicolumn{4}{|c|}{ Manual1/Manual2 } \\
\hline 9NS & $0.12 \pm 0.58$ & $-0.20 \pm 0.89 \quad 0.82^{\mathrm{a}} \pm 0.54$ & $1.04^{\mathrm{a}} \pm 1.19$ \\
\hline $9 \mathrm{~S}$ & $-0.06 \pm 2.50$ & $0.51 \pm 2.06 \quad 0.85 \pm 1.31$ & $0.46 \pm 1.03$ \\
\hline All & $0.03 \pm 1.76$ & $0.15 \pm 1.58 \quad 0.84^{\mathrm{a}} \pm 0.97$ & $0.75 \pm 1.12$ \\
\hline \multicolumn{4}{|c|}{$\begin{array}{ll}0.05 \perp 1.10 & 0.15 \perp 1.50 \quad 0.04+0.91\end{array}$} \\
\hline 9NS & $0.04 \pm 0.98$ & $0.89^{\mathrm{ab}} \pm 0.92-1.06^{\mathrm{ab}} \pm 1.12$ & $0.75 \pm 1.12$ \\
\hline $9 \mathrm{~S}$ & $0.11 \pm 1.12^{\mathrm{c}}$ & $0.24 \pm 1.38-1.39^{\mathrm{ab}} \pm 1.64$ & $1.98^{\mathrm{ab}} \pm 1.11$ \\
\hline All & $0.08 \pm 1.02^{\mathrm{c}}$ & $0.56 \pm 1.18-1.23^{\mathrm{ab}} \pm 1.37$ & $1.37^{\mathrm{a}} \pm 1.25$ \\
\hline \multicolumn{4}{|c|}{ SA/Manual2 } \\
\hline 9NS & $0.15 \pm 0.72$ & $1.13^{\mathrm{ab}} \pm 1.44-0.99^{\mathrm{ab}} \pm 0.79$ & $1.74 \pm 0.62$ \\
\hline $9 \mathrm{~S}$ & $0.31 \pm 2.19$ & $1.02 \pm 1.56-1.23^{\mathrm{b}} \pm 1.94$ & $2.64^{\mathrm{ab}} \pm 1.25$ \\
\hline All & $0.23 \pm 1.58$ & $1.08^{\mathrm{ab}} \pm 1.46-1.11^{\mathrm{ab}} \pm 1.44$ & $2.19^{\mathrm{a}} \pm 1.07$ \\
\hline
\end{tabular}

NS: nonstenotic valves; S: stenotic valves. Results given as $\mu \pm 1.0 \sigma$.

${ }^{\text {a }} p<0.05$, two-tailed paired $t$-test against 0 .

${ }^{\mathrm{b}} p<0.05$, two-tailed paired $t$-test against Manual1/Manual2.

${ }^{\mathrm{c}} p<0.05$, two-tailed $F$-test against Manual1/Manual2.

Results for the SA [24] are also presented.

16 GB of shared memory). Among the framework's three steps, the SAx planes detection algorithm had the highest computational burden $(6.1 \pm 0.7 \mathrm{~s}$, i.e., around $0.3 \mathrm{~s}$ per reference surface used), followed by the automatic initialization procedure ( $4.8 \pm$ $0.8 \mathrm{~s})$ and finally the segmentation step $(1.0 \pm 0.4 \mathrm{~s})$. When considering the individual stages of the initialization algorithm, the 3-D rotation-invariant GHT, the vessel-like dark tube detector, the axis refinement, and the axis endpoints' estimation took, on average, $1.5 \pm 0.4 \mathrm{~s}, 1.6 \pm 0.4 \mathrm{~s}, 1.5 \pm 0.3 \mathrm{~s}$, and $0.2 \pm$ $0.04 \mathrm{~s}$, respectively. Note that, for computational efficiency, a MATLAB-generated C implementation of the GHT and ICP algorithms were wrapped in the MATLAB code.

\section{F. Clinical Measures Evaluation}

Table II presents the agreement between the fully automatically estimated perimeter-derived diameters (FA) and the two experts (for the 18 successfully initialized datasets), assessed at the four levels of interest (LVOT, AoA, SoV, and STJ). Interobserver variability (Manual1/Manual2) is also presented, together with the results for the original semiautomatic approach (SA) [24]. The results are reported separately for nonstenotic and stenotic valves, as well as all together. Moreover, a two-tailed paired $t$-test and $F$-test were used to check for statistically significant differences $(p$-values $<0.05)$ in their biases and limits of agreement, respectively.

Overall, a good agreement was found between the automatic and manual measures. Specifically, similar LOAs were observed (no statistically significant differences compared with the interobserver variability), with the fully automatic strategy showing 


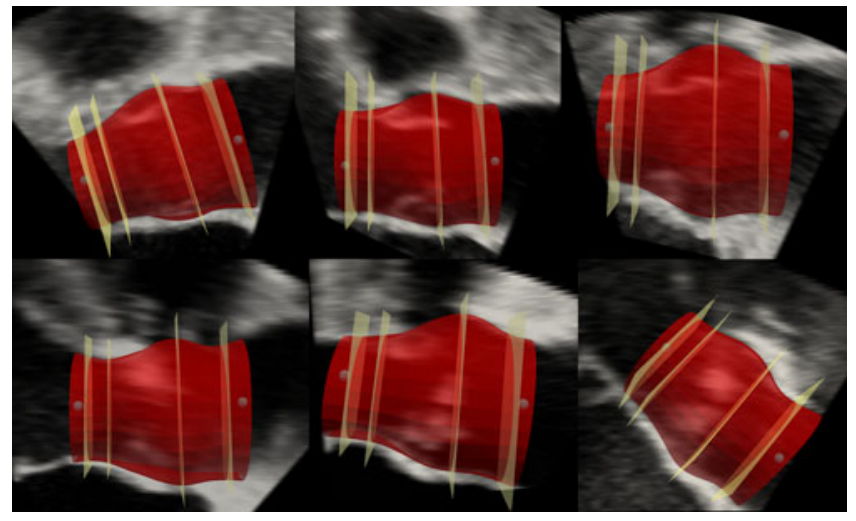

Fig. 9. Fully automatic segmentation results for three nonstenotic (top row) and three stenotic (bottom row) AV datasets (light blue spheres: automatically identified axis endpoints; red surface: segmented AV trunk; yellow planes: automatically extracted SAx planes at the LVOT, AoA, SoV, and STJ levels).

TABLE III

Agreement Between Fully Automatic and Manual Perimeter-Derived Diameters (IN Mm) FOR the Validation Set (GIVEN AS $\mu \pm 1.0 \sigma)$

\begin{tabular}{ccccc}
\hline \hline & LVOT & AoA & SoV & STJ \\
\hline $19 \mathrm{~S}$ & $0.76 \pm 1.69$ & $0.88^{\mathrm{a}} \pm 1.16^{\mathrm{c}}$ & $-1.76^{\mathrm{ab}} \pm 1.98$ & $0.90^{\mathrm{a}} \pm 1.60$ \\
\hline \hline
\end{tabular}

S: stenotic valves; IOV: interobserver variability.

${ }^{a} p<0.05$, two-tailed paired $t$-test against 0

${ }^{\mathrm{b}} p<0.05$, unpaired $t$-test against IOV (with Welch's correction).

${ }^{c} p<0.05$, two-tailed $F$-test against IOV.

a tendency for significant biases at most levels. Contrarily to the manual analysis, no variability exists on the proposed fully automatic framework.

Fig. 9 illustrates fully automatic segmentation results, with corresponding automatically identified axis endpoints and the four relevant SAx planes, for three nonstenotic and three stenotic datasets. Overall, a robust and accurate 3-D segmentation of the AV trunk and a precise extraction of the relevant SAx planes was achieved for the distinct cases.

\section{G. Clinical Validation Experiment}

As a final experiment, the proposed framework was applied to 20 additional stenotic AV cases, with the fully automatic measurements being extracted while blinded to the manual ones. Note that all algorithm parameters were kept the same, therefore assessing the methods' true applicability and generalization to new unseen clinical data.

In this new set of cases, the framework was found to be feasible in $95 \%$, with only one case failing to be initialized automatically due to poor image quality and pronounced dropout artifacts. Table III presents the agreement against expert's measurements at the four levels for the successfully analyzed cases (19 out of 20), together with the comparison against interobserver variability. Interestingly, similar (in some cases, even slightly better) LOAs were found for these new cases, com-

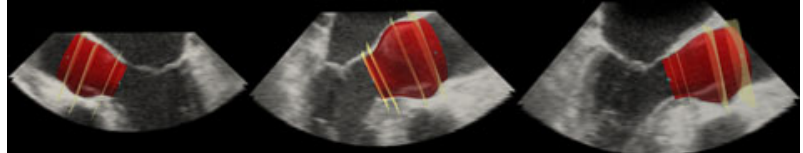

Fig. 10. Example fully automatic initialization and segmentation of three 3-D-TEE acquisitions with large FOVs.

pared wiht the ones obtained for the training/testing set (9S in Table II).

\section{DISCUSSION}

In this study, we sought to evaluate the feasibility, robustness, and accuracy of a novel fully automatic algorithm for AV trunk segmentation in 3-D-TEE datasets of both nonstenotic and stenotic AVs.

The first module of the proposed framework focuses on the identification of the AV axis endpoints (i.e., AscAo and LVOT detection), required to initialize the subsequent automatic segmentation strategy. Hereto, a multistage algorithm was employed, splitting the detection problem into three steps:

1) AV centroid positioning;

2) axis identification (with an additional stage for its refinement); and

3) endpoints estimation.

This procedure allows us to reduce the degrees of freedom (DOF) searched after each step, starting with a 3-D positioning problem, followed by the orientation estimation with 2 DOFs and finally point estimation along the axis found (1 DOF). Note that although consecutive steps are applied, continuous refinement of the estimations in subsequent stages is still permitted (see Table I), thus increasing the method's robustness and accuracy. Ultimately, the endpoints' estimation presented a median $\mathrm{P} 2 \mathrm{Pt}$ distance error of near $3 \mathrm{~mm}$, with a P2Pl error lower than $2 \mathrm{~mm}$ (see Table I).

In terms of feasibility, the proposed module obtained successful results in $90 \%$ of the datasets (Table I), with only two cases presenting visually wrong initializations. Specifically, one failure was related to a low image quality at the LVOT, misleading the fitting of the dark vessel-like tube (i.e., oblique to the true one) and passing at the LVOT level outside the lumen (nonrecoverable by the segmentation strategy). The other case was related to an abnormally lengthy valve (over $40 \mathrm{~mm}$ from AscAo to the LVOT), which when applying the proposed heuristic rule failed to simultaneously place both endpoints successfully (good LVOT positioning but short on locating the AscAo). Nonetheless, in clinical practice, either case could be easily corrected at run-time by asking the user to modify the wrongly positioned axis endpoint. Remarkably, note that the strategy was able to successfully initialize one dataset in which the AV was rotated $90^{\circ}$ from its usual orientation, proving the interest and ability of the proposed rotation-invariant GHT approach. Notice also that, although evaluated for 3-D-TEE-zoomed views only (recommended for TAVI [8]), the proposed method is also applicable for nonzoomed views (see Fig. 10).

Concerning the automatic placement of the relevant SAx planes, the reproducibility test proved the robustness of the 
proposed approach against both initialization and segmentation (see Section III-D1, Fig. 7). Indeed, even for significant displacements of the $\mathrm{AV}$ axis endpoints (3.73 \pm 2.19 and $3.51 \pm$ $2.28 \mathrm{~mm}$ for AscAo and LVOT, respectively, and up to $10 \mathrm{~mm}$ ), the planes' estimation errors presented a median centroid distance and an angular difference lower than $2 \mathrm{~mm}$ and $7^{\circ}$, respectively, for all levels. These results translated into narrow limits of agreement of the automatic measurements extracted (see Fig. 7), particularly when compared with the interobserver variability (see Table II). This low variability is primarily linked to three favorable properties of the segmentation and planes' estimation modules, namely:

1) the ability to partially correct the off-axis errors in the initial endpoints' positioning through the AV axis reinitialization strategy used throughout the segmentation (see [24] for details);

2) the ability to surpass suboptimal longitudinal positioning of the axis endpoints during segmentation, since such variability is embedded in the profile-based SSM used (details in [24]); and

3) the robustness of the ICP algorithm against the initial points, since the full AV shape is used to align segmented and reference surfaces in order to estimate the SAx planes (see Fig. 6).

Importantly, such robustness was kept when considering the fully automatic pipeline (i.e. fusing with the automatic initialization module), with the SAx planes' estimation errors being indistinguishable from those obtained between experts (Figs. 8 and 9).

The agreement with experts' measurements revealed statistically similar LOAs compared with the interobserver variability (see Table II), particularly for stenotic cases. Indeed, although broader LOAs were found for these cases (compared with the nonstenotic ones), also a higher variability between experts was found (see Manual1/Manual2, 9S). Such result shows the increased difficulty in delineating the $\mathrm{AV}$ in these patients, mainly due to the acoustic shadowing artifacts present and the generally lower image quality. When compared with the previous semiautomatic strategy, although slightly wider LOAs were found, these were not statistically significant. Moreover, these LOAs were also competitive to the agreement reported by the state-ofthe-art algorithms in TEE data $(0.62 \pm 1.7$ and $0.46 \pm 2.3 \mathrm{~mm}$ for annulus diameters in stenotic cases [21] and $1.1 \pm 1.3,3.6$ $\pm 2.3,0.5 \pm 1.5$, and $0.2 \pm 1.4$ for sagittal and coronal annulus, SoV and STJ measures in a broad population [33]) and comparable to the reported interobserver variability $(-0.16 \pm 2.1 \mathrm{~mm}$ for annulus diameters in stenotic cases [21] and $1.2 \pm 1.3$ and $2.0 \pm 2.6 \mathrm{~mm}$ against sagittal and coronal annulus measures for a broad population [33]).

Regarding the observed biases, automatic measurements presented larger diameters compared with the manual ones at the LVOT, AoA, and STJ levels, but lower on the sinuses. The latter is partially associated with the fact that the automatic perimeterderived diameters are lower due to the surface's higher smoothing in the regions between bulges. In the literature, similar positive biases are reported for the AoA by other state-of-the-art methodologies [21], [33], [34], and are frequently found when comparing MDCT with TEE measures [35], [36]. This is probably related to the experts' tendency in TEE to delineate at the transition between the lumen and the wall, instead of closer to the more compact bright wall tissue [24].

Importantly, all these results were corroborated when applying the proposed fully automatic framework to 20 additional and previously unseen stenotic cases (see Table III). With a feasibility of 95\%, the method showed narrow LOAs, particularly for the annulus measures. Indeed, the LOAs are within the range of the prosthesis models' sizes (typically $3 \mathrm{~mm}$ apart). Given that the LOAs are the most relevant metric for TAVI prosthesis sizing, it ultimately proves the framework's applicability and robustness to this specific population. In addition, this novel method brings the possibility for a more reproducible analysis in 3-D-TEE data, and may help in increasing its use for TAVI planning. While MDCT is frequently used, ultrasound avoids the use of ionizing radiation and, most importantly, of contrast agents (as these patients often have impaired renal function [3], [10]). Indeed, 3-D-TEE is the only available imaging modality in patients with contraindications to MDCT. Moreover, even centers using MDCT as the primary imaging modality may benefit from precise measurement techniques for ultrasound data. In borderline sizing cases (i.e. in which the measured diameter fits in-between two model sizes), measures taken by other modalities (namely 3-D-TEE), gender or calcification severity and location are paramount for the final sizing decision.

Interestingly, note that the proposed fully automatic methodology mitigates the problem of interobserver variability, while speeding up the analysis. Indeed, the proposed approach requires around $12 \mathrm{~s}$ to automatically quantify the four levels of interest (Section III-E), which would take between 5 and $10 \mathrm{~min}$ in clinical practice. Moreover, if a supervisor approach (i.e., with small manual corrections, namely for minor shifts in the endpoints' positioning or the SAx planes' location) would be employed, the automated strategy is still expected to present a significantly lower interobserver variability (see the results of the original semiautomatic strategy [24] and the reproducibility test in Fig. 7). This was, however, not validated in this study.

Finally, and as observed for the original semiautomatic strategy [24], although here validated for the analysis of mid-systolic frames only (frequently used in practice for TAVI prosthesis sizing [4], [35]), the proposed fully automatic algorithm allows segmenting any cardiac frame in a 3-D-TEE sequence (although not shown in the current experiments). Indeed, there is no assumption on the valve's state, with the only module slightly dependent on it being the dark vessel-like tube detection. Nonetheless, as shown here for the stenotic cases (in which the leaflets are still present in the lumen), the algorithm is able to surpass this limitation.

\section{CONCLUSION}

In this paper, a novel fully automatic AV tract segmentation algorithm for 3-D-TEE datasets was proposed and validated for the quantification of relevant $\mathrm{AV}$ measures. The method showed to be able to robustly and accurately quantify both nonstenotic and stenotic AV with an overall feasibility above $90 \%$. Auto- 
matically extracted measures showed a good agreement with experts' ones, while mitigating the variability induced by experts' manual delineations and significantly reducing the analysis time. In summary, our results show the appeal of the proposed framework for automatic AV segmentation and quantification for TEE-based TAVI planning.

In the future, we intend to validate the proposed algorithm in a larger clinical database, both using a fully automatic pipeline and a supervised approach. Such study might shed light on the interobserver variability when using the latter approach, as well as its real-world influence in the prosthesis sizing.

\section{ACKNOWLEDGMENT}

The authors would like to thank D. Brackman (Philips Healthcare, Belgium) for giving access to the 3-D-TEE voxel data of their system and his logistic support, and Dr. S. Bouchez (UZ Gent, Belgium) for providing the example nonzoomed 3-D-TEE datasets in Fig. 10.

\section{REFERENCES}

[1] A. Vahanian et al., "Guidelines on the management of valvular heart disease (version 2012)," Eur. Heart J., vol. 33, no. 19, pp. 2451-2496, 2012.

[2] J. J. Bax et al., "Open issues in transcatheter aortic valve implantation. Part 1: Patient selection and treatment strategy for transcatheter aortic valve implantation," Eur. Heart J., vol. 35. pp. 2627-2638, 2014.

[3] L. A. Smith et al., "Real-time three-dimensional transesophageal echocardiography adds value to transcatheter aortic valve implantation," J. Amer Soc. Echocardiography, vol. 26, no. 4, pp. 359-369, 2013.

[4] F. A. Flachskampf et al., "Recommendations for transoesophageal echocardiography: EACVI update 2014," Eur. Heart J.-Cardiovascular Imag., vol. 11, pp. 557-576, 2014.

[5] R. T. Hahn et al., "Guidelines for performing a comprehensive transesophageal echocardiographic examination: Recommendations from the American Society of Echocardiography and the Society of Cardiovascular Anesthesiologists," J. Amer. Soc. Echocardiography, vol. 26, no. 9, pp. 921-964, 2013.

[6] R. T. Hahn et al.,"Recommendations for comprehensive intraprocedural echocardiographic imaging during TAVR," JACC, Cardiovascular Imag., vol. 8, no. 3, pp. 261-287, 2015.

[7] F. Flachskampf et al., "Recommendations for transoesophageal echocardiography: Update 2010," Eur. Heart J.-Cardiovascular Imag., vol. 11, no. 7 , pp. $557-576,2010$.

[8] J. Zamorano et al., "The use of imaging in new transcatheter interventions: An eacvi review paper," Eur. Heart J. Cardiovascular Imag., vol. 17, no. 8, pp. 835-835af, 2016.

[9] G. Tamborini et al., "Feasibility and accuracy of 3D-TEE versus CT for the evaluation of aortic valve annulus to left main ostium distance before transcatheter aortic valve implantation," JACC, Cardiovascular Imag., vol. 5, no. 6, pp. 579-588, 2012.

[10] O. K. Khalique et al., "Aortic annular sizing using a novel 3-dimensional echocardiographic method use and comparison with cardiac computed tomography," Circulation, Cardiovascular Imag., vol. 7, no. 1, pp. 155 $163,2014$.

[11] V. C.-C. Wu et al., "Aortic root geometry in patients with aortic stenosis assessed by real-time three-dimensional transesophageal echocardiography," J. Amer. Soc. Echocardiography, vol. 27, no. 1, pp. 32-41, 2014.

[12] R. I. Ionasec et al., "Patient-specific modeling and quantification of the aortic and mitral valves from 4-D cardiac CT and TEE," IEEE Trans. Med. Imag., vol. 29, no. 9, pp. 1636-1651, Sep. 2010.

[13] I. Wächter et al., "Patient specific models for planning and guidance of minimally invasive aortic valve implantation," in Proc. Med. Image Comput. Comput.-Assisted Intervention Conf., 2010, pp. 526-533.

[14] V. Delgado et al., "Automated assessment of the aortic root dimensions with multidetector row computed tomography," Ann. Thoracic Surgery, vol. 91, no. 3, pp. 716-723, 2011.
[15] Y. Zheng et al.. "Automatic aorta segmentation and valve landmark detection in C-arm CT for transcatheter aortic valve implantation," IEEE Trans. Med. Imag., vol. 31, no. 12, pp. 2307-2321, Dec. 2012.

[16] M. Elattar et al., "Automatic segmentation of the aortic root in CT angiography of candidate patients for transcatheter aortic valve implantation," Med. Biol. Eng. Comput., vol. 52, no. 7, pp. 611-618, 2014.

[17] J. K. Swee and S. Grbić, "Advanced transcatheter aortic valve implantation (TAVI) planning from CT with ShapeForest," in Medical Image Computing and Computer-Assisted Intervention-MICCAI 2014. Berlin, Germany: Springer-Verlag, 2014, pp. 17-24.

[18] Y. Nie et al., "A novel aortic valve segmentation from ultrasound image using continuous max-flow approach," in Proc. IEEE 2013 35th Annu. Int. Conf. Eng. Med. Biol. Soc., 2013, pp. 3311-3314.

[19] B. Dong et al., "Aortic valve segmentation from ultrasound images based on shape constraint CV model," in Proc. IEEE 2013 35th Annu. Int. Conf. Eng. Med. Biol. Soc., 2013, pp. 1402-1405.

[20] J. Cai et al., "Real-time aortic valve segmentation from transesophageal echocardiography sequence," Int. J. Comput. Assisted Radiol. Surg., vol. 10, no. 4, pp. 447-458, 2015.

[21] J. Bersvendsen et al., "Automatic measurement of aortic annulus diameter in 3-dimensional transoesophageal echocardiography," BMC Med. Imag., vol. 14, no. 1, 2014, Art. no. 31.

[22] F. M. Weber et al., "Analysis of mitral valve motion in 4D transesophageal echocardiography for transcatheter aortic valve implantation," in Statistical Atlases and Computational Models of the Heart-Imaging and Modelling Challenges. Berlin, Germany: Springer-Verlag, 2015, pp. 168-176.

[23] A. M. Pouch et al., "Medially constrained deformable modeling for segmentation of branching medial structures: Application to aortic valve segmentation and morphometry," Med. Image Anal., vol. 26, no. 1, pp. 217-231, 2015

[24] S. Queiros et al., "Aortic valve tract segmentation from 3D-TEE using shape-based B-spline explicit active surfaces," IEEE Trans. Med. Imag., vol. 35, no. 9, pp. 2015-2025, 2016.

[25] S. Queirós et al., "Fast automatic myocardial segmentation in 4D cine CMR datasets," Med. Image Anal., vol. 18, no. 7, pp. 1115-1131, 2014.

[26] D. Ballard, "Generalizing the hough transform to detect arbitrary shapes," Pattern Recognit., vol. 13, no. 2, pp. 111-122, 1981

[27] O. Ecabert et al., "Automatic model-based segmentation of the heart in CT images," IEEE Trans. Med. Imag., vol. 27, no. 9, pp. 1189-1201, Sep. 2008

[28] A. A. Rozhentsov et al., "Modified generalized hough transform for 3D image processing with unknown rotation and scaling parameters," Optoelectron., Instrum. Data Process., vol. 49, no. 2, pp. 131-141, 2013.

[29] J. Canny, "A computational approach to edge detection," IEEE Trans. Pattern Anal. Mach. Intell., vol. PAMI-8, no. 6, pp. 679-698, Nov. 1986.

[30] A. F. Frangi et al., "Multiscale vessel enhancement filtering," in Medical Image Computing and Computer-Assisted Intervention-MICCAI 1998. Berlin, Germany: Springer, 1998, pp. 130-137.

[31] O. Bernard et al., "Standardized evaluation system for left ventricular segmentation algorithms in 3D echocardiography," IEEE Trans Med Imag. vol. 35, no. 4, pp. 967-977, Apr. 2015.

[32] J. M. Bland and D. Altman, "Statistical methods for assessing agreement between two methods of clinical measurement," Lancet, vol. 327, no. 8476, pp. 307-310, 1986.

[33] A. Calleja et al., "Automated quantitative 3-dimensional modeling of the aortic valve and root by 3 -dimensional transesophageal echocardiography in normals, aortic regurgitation, and aortic stenosis comparison to computed tomography in normals and clinical implications," Circulation, Cardiovascular Imag., vol. 6, no. 1, pp. 99-108, 2013.

[34] A. García-Martín et al., "Accuracy and reproducibility of novel echocardiographic three-dimensional automated software for the assessment of the aortic root in candidates for thanscatheter aortic valve replacement," Eur. Heart J.-Cardiovascular Imag., 2015, Art. no. jev238.

[35] B. Vaquerizo et al., "Three-dimensional echocardiography vs. computed tomography for transcatheter aortic valve replacement sizing," Eur. Heart J. Cardiovascular Imaging, pp. 1-9, 2015.

[36] H. Wang et al., "Comparison of aortic annulus size by transesophageal echocardiography and computed tomography angiography with direct surgical measurement," Amer. J. Cardiol., vol. 115, no. 11, pp. 1568-1573, 2015

Authors' photographs and biographies not available at the time of publication. 